cell carcinoma and 2 benign diseases. SEMS deployment was successful in all 110 procedures. Of the 46 traversable tumours, 22 underwent ET and 24 FT for SEMS placement. 64 patients had non traversable tumours (with standard endoscope). HT was used in 21 and FT in 43 patients. Length of the stent deployed was not statistically different in both groups (HT $11.4 \pm 0.5$ $\mathrm{cm}$, FT $11.0 \pm 0.5 \mathrm{~cm}, \mathrm{P}=0.98)$. Good dysphagia alleviation was seen in both groups (dysphagia scores, HT cohort pre-stent $2.7 \pm 0.16$, post-stent $0.53 \pm 0.87 \mathrm{P}<0.001$; FT cohort prestent $2.53 \pm 0.09$, post-stent $0.44 \pm 0.11 \mathrm{P}<0.001$ ). Cumulative complications (reflux, pain, bleeding, food bolus obstruction) were similar for both techniques (HT $0.28 \pm 0.1$, FT 0.34 $\pm 0.1, \mathrm{P}=0.62)$. There was a tendency towards higher re-intervention for tumour overgrowth in the FT cohort (FT 7/43 patients, $129.4 \pm 35.5$ days; HT 2/21 patients, $215 \pm 60$ day, $\mathrm{P}$ $=0.33)$. Neither the stent length $(\mathrm{P}=0.89)$ nor the technique used, had an influence on the need for re-stenting for tumour overgrowth $(\mathrm{P}=0.68)$. Median survival was 141 days (IQR 46180 ) in the HT group and 121 days (IQR 27.75-188.5) in the FT group. There were no instances of stent migration in the non-traversable group.

Conclusion Outcomes following SEMS insertion in both techniques were similar. Hybrid approach is an acceptable alternative to fluoroscopy only, in patients with non-traversable tumours, with the added advantage of more accurate positioning of the proximal end of the stent under direct endoscopic visualisation. Limitations of the study are its retrospective nature, lack of data on diameter of stents.

Disclosure of Interest None Declared.

\section{PTU-016 WIRELESS CAPSULE ENDOSCOPE LOCALISATION BASED ON VISUAL ODOMETRY}

${ }^{1} \mathrm{~A}$ Koulaouzidis*, ${ }^{2} \mathrm{DK}$ lakovidis, ${ }^{2}$ E Spyrou. ${ }^{1}$ The Royal Infirmary of Edinburgh, Edinburgh, UK; ${ }^{2}$ Technological Educational Institute of Central Greece, Lamia, Greece

\subsection{6/gutjnl-2014-307263.90}

Introduction The localisation of a wireless capsule endoscope (WCE) within the small-bowel is typically performed by wearable radiofrequency sensors triangulation. The accuracy of this approach is low. ${ }^{1}$ Only a few approaches have been proposed for WCE localisation based on visual features. These include methods addressing the estimation of the rotation angle of the capsule $^{2,3}$ and temporal video segmentation methods. ${ }^{4}$ We present a WCE localisation method, based only on visual information extracted from conventional WCE recording.

Methods Automatic detection of points of interest (POI) in WCE video frames, matching of the detected POI between consecutive frames, and determination of actual correspondences between subsets of these POI based on the random sample consensus (RANSAC) algorithm was performed. Maximally stable extremal regions (MSER) algorithm, instead of the speeded up feature extraction (SURF) algorithm, was used. Based on the scaling and the rotation of the content of the consecutive WCE frames, it is possible to estimate the displacement and the rotation of the capsule within the GI tract. For the ex-vivo experiment; a standard simulated intestinal environment was created. Markers were sewn (at set, pre-recorded distances) onto the luminal surface of porcine small-bowel through which a capsule (MiroCam ${ }^{\circledR}$, IntroMedic Co Ltd, Seoul, Korea) was propelled. Results Comparative experiments using both SURF and MSER features, which indicated the superiority of the former over the latter, we conducted. We worked on a corpus of 1070 WCE frames (634 indicating forward motion, 436 indicating backward motion). The accuracy using SURF features was $81.5 \%(87.2 \%$ on forward motion, $73.2 \%$ on backward motion), while using MSER was $67.2 \%$ (79.8\% on forward motion, and $48.9 \%$ backward motion). Noteworthy, the proposed algorithm often fails when using MSER $(6.7 \%$ of frames while $<0.1 \%$ when using SURF) and a transform is not estimated due to the lack of adequate correspondences between interest points.

Conclusion Visual odometry is a promising technique and - potentially - a feasible alternative to other localisation approaches in WCE.

\section{REFERENCES}

1 Than TD, et al. A review of localization systems for robotic endoscopic capsules. IEEE Transactions on Biomedical Engineering 2012;59:2387-99

2 Spyrou E, lakovidis D. Homography-based orientation estimation for capsule endoscope tracking. in 2012 IEEE Int. Conf. on Imaging Systems and Techniques (IST), IEEE, 2012:101-105

3 Mackiewicz $M$, et al. Wireless capsule endoscopy color video segmentation. IEEE Transactions on Medical Imaging 2008;27:1769-81

4 Spyrou E, lakovidis DK. Video-based measurements for wireless capsule endoscope tracking. Measurement Science and Technology, Measurement Science and Technology, IOP Publishing, 2014;25:015002 (14pp)

Disclosure of Interest None Declared.

\section{PTU-017 THE USE OF SMALL BOWEL CAPSULE ENDOSCOPY IN THE OCTOGENARIANS GROUP; THE EDINBURGH EXPERIENCE}

L Bartzis, A Koulaouzidis*. The Royal Infirmary of Edinburgh, Edinburgh, UK

\subsection{6/gutjnl-2014-307263.91}

Introduction Over the last 13 years, the clinical use of capsule endoscopy (CE) has revolutionised the investigation pathways for the small-bowel. Although non-invasive (as procedure), there are reports of capsule aspiration in certain patient-groups. ${ }^{1}$ Moreover, CE video review is a time-consuming process and - on occasions - with limited diagnostic yield (DY). There is scarcity of data on the use of CE in octogenarians. ${ }^{2-4}$ We aim to report our centre experience in using $\mathrm{CE}$ in octogenarians.

Methods Retrospective study; the SBCE data base of our unit was interrogated for patients $>80$ years of age who underwent CE. Categorical data are reported as mean \pm SD. The Fischer's exact, the chi-square and the $t$ (unpaired) tests were used to compare datasets. A two-tailed $P$ value of $<0.05$ was considered statistically significant.

Results 1,477 patients underwent small-bowel CE between 2005 and 2013. $93 \mathrm{CE}$ were performed in $84(35 \mathrm{M} / 59 \mathrm{~F})$ octogenarians; mean age $84 \pm 2.9$ years. PillCam ${ }^{\circledR} \mathrm{SB} 1 / \mathrm{SB} 2$ and MiroCam ${ }^{\circledR}$ were used in 61 and $32 \mathrm{CE}$ examinations, respectively. Ten (11.9\%) patients had more than $1 \mathrm{CE}$. One patient was unable to swallow the capsule, and in another the capsule was retained in the stomach. The $\mathrm{CE}$ report was unavailable in one case. Indications for small-bowel CE were IDA: 44, OGIB: 29, OBIG+IDA: 6, diarrhoea 4, small-bowel varices: 1. Forty-five $(53.6 \%)$ patients subsequently died. The mean time from SBCE to death was 23 months (SD 20.9, range: 0.13-83 months).

The DY (all findings) of CE in our octogenarian cohort was $56.8 \%$. Vascular lesions (any P class)/active bleeding were found in 33 , inflammatory pathology in 9 , and other in 4 CE. No neoplastic pathology was identified. The DY was independent to the indications for the procedure $(P=0.166)$, the small-bowel CE system used $(P=0.068)$, the patient final outcome i.e. deceased/ alive $(P=0.051)$ and/or the time from $C E$ to death $(P=0.053)$. 
Conclusion CE in patients $>80$ years of age has high DY, but sinister pathology in this cohort is rare. Furthermore, smallbowel CE has limited impact on the final patient outcome in this patient group.

\section{REFERENCES}

1 Koulaouzidis A, et al. Small-bowel capsule endoscopy: a ten-point contemporary review. World J Gastroenterol 2013;19:3726-46

2 Koulaouzidis A, et al. The use of small-bowel capsule endoscopy in iron-deficiency anemia alone; be aware of the young anemic patient. Scand I Gastroenterol 2012;47:1094-100

3 Tsibouris $\mathrm{P}$, et al. Capsule endoscopy findings in patients with occult or overt bleeding older than 80 years. Dig Endosc 2012;24:154-8

4 Sidhu R, McAlindon ME. Age should not be a barrier to performing capsule endoscopy in the elderly with anaemia. Dig Dis Sci 2011;56:2497-8

Disclosure of Interest None Declared.

\section{PTU-018 AUTOMATIC DETECTION OF 'SUSPICIOUS' CAPSULE ENDOSCOPY VIDEO SEGMENTS}

${ }^{1} \mathrm{~A}$ Koulaouzidis*, ${ }^{2} \mathrm{~S}$ Tsevas, ${ }^{2} \mathrm{DK}$ lakovidis. ${ }^{1}$ The Royal Infirmary of Edinburgh, Edinburgh, UK; ${ }^{2}$ Technogical Educational Institute of Central Greece, Lamia, Greece

\subsection{6/gutinl-2014-307263.92}

Introduction Manual review and annotation of a capsule endoscopy (CE) video requires a considerable amount of reviewing time. The diagnostic accuracy of this process may decrease over time due to reviewer tiredness. Recent studies showed an average detection rate - for the clinically significant findings - as low as $40 \%{ }^{1}$ We present a generic computational framework for automatic detection of abnormalities in $\mathrm{CE}$ videos.

Methods A CE video (MiroCam ${ }^{\circledR}$, InrtoMedic Co Ltd, Seoul, Korea), depicting inflammatory changes (aphthae, mucosal breaks, ulcers, erythema) was reviewed and manually annotated by experienced CE reviewer. A total of 1984 frames, containing any pathology, were thumbnailed. The proposed framework considers video frames as members of a vector space represented by their colour information. An unsupervised data reduction algorithm, ${ }^{2}$ which does not require any prior knowledge about the data, was then applied on each segment. This algorithm clusters together frames that exhibit similar characteristics e.g., colour distributions. Its output is a subset of video frames extracted from each cluster by applying a threshold to the clustering result. The extracted frames are characteristic of the particular video segment and as a result representative of possible lesions.

Results The evaluation of the proposed framework aimed to determine its accuracy, in terms of the ratio of the neighbourhoods represented by at least one frame in the system's output and the neighbourhoods that were manually annotated as suspicious for containing lesions. The parameters considered include clustering from 2 to 6 clusters and thresholds ${ }^{2}$ varying from 0.004 to 0.6 . The obtained accuracy ranged between $76 \%$ to $98 \%$ depending on the desired sensitivity level of the algorithm, controlled by the threshold. Furthermore, the automatic selection of the representative CE video segments performed by the proposed approach, the number of video frames to be thoroughly examined can be reduced from $30 \%$ to $60 \%$ of the original video, depending on the clustering and threshold settings.

Conclusion The application of the proposed framework to the evaluation of $\mathrm{CE}$ videos may reduce the rate of false negative evaluations by attracting the attention of the reviewer to automatically identified video segments (or single frames) of interest which are likely to contain lesions.

\section{REFERENCES}

1 Zheng Y, Hawkins L, Wolff J, Goloubeva O, Goldberg E. Detection of lesions during capsule endoscopy: physician performance is disappointing. Am J Gastroenterol. 2012;107:554-60

2 lakovidis DK, Tsevas S, Polydorou A. Reduction of capsule endoscopy reading times by unsupervised image mining. Comput. Med. imaging Graph. Off. J. Comput. Med. Imaging Soc. 2010;34(6):471-478

Disclosure of Interest None Declared.

\section{PTU-019 ANTITHROMBOTIC VS. ULCER EFFECTS IN NON-VARICEAL BLEEDING IN USERS OF ANTITHROMBOTIC DRUGS}

${ }^{1,2}$ AS Taha*, ${ }^{2} \mathrm{C}$ McCloskey, ${ }^{2} \mathrm{~T}$ Craigen, ${ }^{1}$ WJ Angerson. ${ }^{1}$ School of Medicine, University of Glasgow, Glasgow, UK; ${ }^{2}$ Gastroenterology, University Hospital Crosshouse, Kilmarnock, UK

\subsection{6/gutjnl-2014-307263.93}

Introduction The use of antithrombotic drugs (ATDs) remains a considerable challenge in the aetiology and management of nonvariceal upper gastrointestinal bleeding (NVUGIB). In the upper gastrointestinal tract, ATD use may result in bleeding by mucosal damage (ulcer effect) or through its basic antithrombotic effect. The clinical significance of these effects is unclear.

In this controlled analysis, we AIMED to clarify the significance of the antithrombotic effect as compared with the ulcer effect in patients with NVUGIB using ATDs.

Methods We previously found that ATD users tended to be older and to have higher comorbidity and different endoscopy findings. To overcome these confounding factors, we compared 202 patients with NVUGIB using ATDs (ATD Group) with 202 patients with NVUGIB but not using ATDs (Controls), having matched both groups in a pairwise manner for age, Charlson comorbidity score and a composite endoscopic score covering the oesophagus, stomach, and duodenum. Antithrombotic drugs included low-dose aspirin, clopidogrel, dipyridamole, warfarin, and heparin. Patients using NSAIDs were excluded. Characteristics of the groups were compared using the Wilcoxon signed rank test and McNemar's test. Continuous variables are reported as median (IQR).

Results The characteristics of the two matched study groups are shown in Table 1.

Conclusion After matching for age, comorbidity, and composite endoscopy score, patients with NVUGIB and using ATDs had significantly lower haemoglobin level, higher Blatchford risk score, and were 1.5 times more likely to be transfused. These effects are most likely to be due to the antithrombotic activity of

\begin{tabular}{|c|c|c|c|}
\hline & ATD $(n=202)$ & Control $(n=202)$ & P-value \\
\hline Age, years, median (IQR) & $72(60-79)$ & $71(60-79)$ & Equal by design \\
\hline Charlson comorbidity score & $1(1-2)$ & $1(1-2)$ & Equal by design \\
\hline Endoscopy score & $1(0-2)$ & $1(0-2)$ & Equal by design \\
\hline Males & $125 / 202(62 \%)$ & $105 / 202(52 \%)$ & 0.06 \\
\hline Smoking & $53 / 200(27 \%)$ & $55 / 199(28 \%)$ & 1.00 \\
\hline Haemoglobin, g/dl & $10.3(8.0-12.8)$ & $12.2(9.4-13.8)$ & 0.02 \\
\hline Urea, mmol/l & $9.8(6.1-14.2)$ & $7.9(5.5-12.5)$ & 0.04 \\
\hline Blatchford score & $8(4-11)$ & $5(2-9)$ & $<0.001$ \\
\hline Transfusion & $79 / 200(40 \%)$ & $52 / 198(26 \%)$ & 0.006 \\
\hline
\end{tabular}

